\title{
The Association Between Low Serum Cholesterol and Non-Cardiovascular Mortality among Italian Males and Females: A Nine- Year Prospective Cohort Ctudy
}

\author{
Samira Taravatmanesh ${ }^{1}$, Nader Parsa ${ }^{1 *}$, Maurizio Trevisan ${ }^{2}$, Pari Mahleaghae \\ Zaheri $^{3}$
}

\begin{abstract}
Background: No study to date provides evidence suggesting that lower cholesterol is associated with excess death in non-cardiovascular disease (NCVD). This study aimed to determine the association between low cholesterol level and NCVD mortality. Methods: A nine-year cohort study was conducted on 3,079 male and 26,005 female Italians aged 20-69 years old. The Cox proportional hazard models implied a hazard ratio with $95 \%$ confidence interval for association. Results: Among males, there were significant inverse associations between the lowest cholesterol decile $(<160 \mathrm{mg} / \mathrm{dl})$ hazard ratio and all-cause deaths and non-cardiovascular deaths, 1.50 (1.19-1.89) and 2.06 (1.54-2.74), respectively. Among females, there was a significant inverse association of lowest and fourth cholesterol deciles, 1.53 (1.01-2.34); 1.52 (1.06-2.18) hazard ratio for all-cause deaths and risk for non-cardiovascular deaths in the same deciles $1.52(0.91-2.50) ; 1.78$ (1.16-2.71), respectively. Remarkably, in depth analysis for NCVD, found significant inverse associations hazard of cholesterol $<160 \mathrm{mg} / \mathrm{dl}$ for cancer, non-cancer liver dysfunction (NCLD), other non-cancer-nonCVD in males and only NCLD death was significant in females. Conclusion: Among males, there were significant inverse hazard associations between the lowest cholesterol decile and all-cause and non-CVD deaths . Among females, there were significant inverse hazard associations of lowest and fourth cholesterol decile for all-cause and also risk first and fourth deciles for non-CVD mortality.
\end{abstract}

Keywords: Low cholesterol- non-cardiovascular- mortality

Asian Pac J Cancer Prev, 20 (5), 1361-1368

\section{Introduction}

Although the mechanism causing low serum cholesterol in acute and chronic illness has not been established yet, parenteral administration of lipopolysaccharide or cytokine mediators of inflammation experimentally reduces cholesterol levels (Auerbach and Parks, 1989). The controversial inverse association has stimulated an examination of cholesterol and non-coronary mortality associations, but the literature is sparse and the conclusions are not robust. The effects of lowering cholesterol with medications on total mortality and death attributable to coronary heart disease (CHD), non-CHD, stroke, non-CVD, cancer, and non-illness mortality (NIM), predominantly accidents, violence, and suicides have been investigated in several primary prevention studies(Kim et al., 2014; Krycer and Brown, 2013; Tanaka and Okamura, 2012).

Longitudinal cohort studies and clinical trials found a significant risk associated with low serum cholesterol and non-CVD mortality (Parsa et al., 2017). These associations were due to preclinical conditions because the inverse association was attenuated or disappeared by excluding early death (Cambien etal., 1980). However, other studies demonstrated that preclinical effects were not the entire explanation for the association between low serum cholesterol and non-CVD related deaths (Parsa et al., 2018). A higher mortality rate of non-cardiovascular, non-cancer causes was also noted in observational studies (Sahebzamani et al., 2013) and among treated cohorts in several cholesterol lowering trials (Holme, 1991). These observations were examined, using a meta-analyses of 32 randomized clinical trials and 19 cohort studies, in the 1990 National Heart, Lung, and Blood Institute of National Institute of Health (NIH) Conference (Jacobs et al., 1992). No study to date has shown that lowering cholesterol extends overall survival. A number of observational cohort studies in a variety of settings found that all-cause

${ }^{1}$ Cardiovascular Research Center, ${ }^{3}$ Shiraz University of Medical Sciences, Shiraz, Iran, ${ }^{2}$ City College of New York ("CCNY"), Provost and Senior Vice President for Academic Affairs, Dean of Medical School, New York. *For Correspondence: drnaderparsabe930@gmail.com 
mortality has a "U", "J", or "L" shaped relationship to serum cholesterol (Bowe et al., 2016; Hasegawa et al., 2016). Therefore, this study aimed to determine if low serum cholesterol $(<160 \mathrm{mg} / \mathrm{dl})$ is a risk factor for non- CVD mortality.

\section{Materials and Methods}

\section{Study population}

This longitudinal study was conducted on 64,957 Italian patients and examined 19 out of 52 risk factors and life expectancy (RIFLE) cohort studies using a 9-year follow up as the endpoint.

\section{Sample size}

There were 30,179 males and 26,005 females aged 20-69 years (Figure 1). Underlying causes of death were coded based on the International Classification of Diseases, 10th revision (World Health Organization, 2010).

\section{Baseline measurements}

Total serum cholesterol was measured in patients' blood samples drawn after a 12-hour fast. Several automated enzymatic methods were employed by all involved laboratories based on WHO Regional Lipid Reference Centre introduced in Prague (Verdery and Goldberg, 1991). Using a calibrated sphygmomanometer, blood pressure was measured in the right arm while the patient was in a sitting position and had taken a four-minute rest, . Observers were trained and tested according to WHO manual (Auerbach and Parks, 1989). Cassettes developed by the London School of Hygiene and the Laboratory of Physiologic Hygiene, University of Minnesota, were used (Ettinger et al., 1990). Systolic and diastolic-2 (5th phase) levels were recorded for analysis, although diastolic-1 was only recorded in most studies. Weight was measured in light underwear, rounded off to the nearest kilogram and height was measured without shoes, expressed in centimeters, following the rules suggested in the WHO manual (Auerbach and Parks, 1989). Smoking status was assessed by a questionnaire directly derived from the WHO cardiovascular survey method manual (Auerbach and Parks, 1989). Age was measured, using the difference between the year of examination and the year of birth, accepting an average error of +6 months. Relative body-mass-index (BMI) was calculated as a percent deviation of actual weight to standard weight based on the mean of body weight distributions by height.

\section{Outcome}

Mortality rates were calculated by gender, based upon decile cholesterol levels in equal intervals from $<160 \mathrm{mg} / \mathrm{d}(<4.4 \mathrm{mmol})$ to $>276 \mathrm{mg} / \mathrm{dl}$ $(>7.14 \mathrm{mmol})$. Gender-specific rates were age-adjusted to the age distribution 30,179 males and 26,005 females, respectively. Cause-specific mortality was determined by non-CVD according to the ICD-10 code (cancer: 140-239, non-cancer liver dysfunction:570-573, accidental-death: 800-829, violence:830-999, and
other-non-CVD: 1-139, 240-389, 460-569, 574-799).

\section{Statistical analysis}

The baseline characteristics were presented as frequencies and mean $\pm \mathrm{SD}$. The Cox proportional hazard models implied a hazard ratio with $95 \%$ confidence interval for association. Reliability and validity of the utilized data were as complete as possible. To obtain complete information regarding cholesterol levels, mortality, and verification of information, variables (e.g. socio-demographic, anthropometric, clinical, laboratory, age, smoking status, BMI, systolic and diastolic pressure) were analyzed for the purpose of controlling confounding or interaction effects. Gender-specific Hazard Ratio and 95\% confidence interval (CI) for death were calculated with reference to the risk of persons with cholesterol levels of $>276 \mathrm{mg} / \mathrm{dl}$. These estimates were adjusted for age and other potential confounders using the Cox proportional hazard models. Due to small sample size for accident, suicide in different cholesterol level deciles, it was not possible for multivariate analysis.

\section{Results}

The mean and standard deviation of body-mass index (BMI), age (year), systolic and diastolic blood pressures $(\mathrm{mm} / \mathrm{Hg})$, serum cholesterol level $(\mathrm{mg} / \mathrm{dl})$, smoking status (never-ex-and current-smoker), and follow-up status (months) were described for males and females according to various deciles. The baseline characteristics of participants within and between each deciles were similar for both gender (Table 1).

According to Table 2, $4.76 \%(n=1,439)$ of men and $1.79 \%(n=467)$ of women died in the total study population. In general, the results revealed an approximately 3 times higher mortality rate among males compared to females. Table 2 also clarifies the specific cause of death in males compared to females with respect to cholesterol level deciles. Leading causes of mortality in males with the lowest cholesterol level were total non-CVD (5.35\%), cancer $(3.03 \%)$, non-cancer liver dysfunction $(1.36 \%)$, violence $(0.22 \%)$, and other non-CVD/non- cancer $(0.69 \%)$. Results indicated that death rates gradually decreased when cholesterol levels increased in different deciles, $5.35 \%$ to $2.60 \%$ in total non-CVD, $3.03 \%$ to $1.85 \%$ in cancer, $1.36 \%$ to $0.14 \%$ in non-cancer liver dysfunction, $0.22 \%$ to $0.09 \%$ in violence, and $0.69 \%$ to $0.44 \%$ o in other non-CVD, respectively. Based on Table 2, males mortality rates compared to females were almost 3.5 times higher for total non-CVD (5.35 vs. 1.60), 5.05 times higher for cancer (3.03 vs. 0.60), 2.06 times higher for non-cancer liver dysfunction (1.36 vs.0.66), 7.33 times higher for violence ( 0.22 vs. 0.03$)$, and 3.45 times higher for other non-CVD/non-cancer ( 0.69 vs. 0.20 ) in the first cholesterol decile. Females age-adjusted specific cause of death rates fluctuated in other deciles of cholesterol. In males, $(1,439)$ with $100 \%$ all-causes of death, $61.3 \%$ (882) were non-CVD and 38.7\% (557) CVD. These results clearly indicated nearly two times higher death rates for non-CVD compared to CVD. In addition, considering all-causes of death $(n=151)$ in the 
DOI:10.31557/APJCP.2019.20.5.1361

Low Serum Cholesterol and Non-Cardiovascular Mortality in Male and Female Population

Table 1. Baseline Characteristics for Male $(n=30179)$ Compared to Female $(n=26,005)$ Population Based upon Decile Cholesterol Level

\begin{tabular}{|c|c|c|c|c|c|c|c|c|c|c|}
\hline \multirow[b]{2}{*}{ 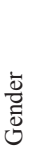 } & \multirow{2}{*}{$\begin{array}{l}\text { Cholesterol } \\
\text { Level } \\
(\mathrm{mg} / \mathrm{dl})\end{array}$} & \multicolumn{4}{|c|}{ Mean \pm SD } & \multicolumn{3}{|c|}{ Smoking Status } & \multirow{2}{*}{$\begin{array}{l}\text { Follow- } \\
\text { up status } \\
\text { (months) }\end{array}$} & \multirow{2}{*}{$\begin{array}{c}\text { Total } \\
\text { sub - } \\
\text { population }\end{array}$} \\
\hline & & BMI* & $\begin{array}{l}\text { Age } \\
\text { (year) }\end{array}$ & $\begin{array}{l}\text { Systolic blood } \\
\text { pressure }(\mathrm{mm} / \\
\mathrm{Hg})\end{array}$ & $\begin{array}{c}\text { Diastolic blood } \\
\text { pressure }(\mathrm{mm} / \\
\mathrm{Hg})\end{array}$ & $\begin{array}{l}\text { Never } \\
(\%)\end{array}$ & $\begin{array}{c}\text { EX- } \\
\text { smoker }(\%)\end{array}$ & $\begin{array}{c}\text { Current } \\
(\%)\end{array}$ & & \\
\hline \multirow{11}{*}{$\stackrel{\frac{0}{\pi}}{\Sigma}$} & $<160$ & $24.98 \pm 3.65$ & $42.45 \pm 13.82$ & $128.72 \pm 19.01$ & $80.74 \pm 11.32$ & $27.5(775)$ & $24.2(682)$ & $48.3(1363)$ & $86.58 \pm 34.28$ & 2,820 \\
\hline & $160-174$ & $25.63 \pm 3.66$ & $44.51 \pm 12.98$ & $130.62 \pm 18.27$ & $82.20 \pm 10.91$ & $26.5(640)$ & $26.9(647)$ & $46.6(1122)$ & $86.26 \pm 32.58$ & 2,409 \\
\hline & $175-186$ & $25.81 \pm 3.51$ & $45.56 \pm 12.64$ & $132.13 \pm 19.50$ & $83.17 \pm 11.28$ & $27.7(714)$ & $26.8(691)$ & $45.5(1171)$ & $85.35 \pm 32.78$ & 2,576 \\
\hline & $187-198$ & $26.04 \pm 3.56$ & $46.66 \pm 11.99$ & $133.32 \pm 19.55$ & $83.55 \pm 11.27$ & $24.4(726)$ & $26.9(802)$ & $48.7(1450)$ & $85.86 \pm 32.43$ & 2,978 \\
\hline & $199-209$ & $26.13 \pm 3.47$ & $47.16 \pm 11.65$ & $133.74 \pm 19.43$ & $84.22 \pm 11.00$ & $25.4(757)$ & $29.3(876)$ & $45.3(1351)$ & $84.49 \pm 32.82$ & 2,984 \\
\hline & $210-221$ & $26.40 \pm 3.51$ & $48.00 \pm 11.42$ & $135.37 \pm 19.67$ & $85.10 \pm 11.13$ & $24.2(797)$ & 28.7 (943) & $47.1(1547)$ & $84.05 \pm 30.87$ & 3,287 \\
\hline & $222-234$ & $26.55 \pm 3.40$ & $48.61 \pm 10.97$ & $136.36 \pm 19.70$ & $85.65 \pm 11.46$ & $22.5(713)$ & $29.6(939)$ & $47.9(1518)$ & $85.86 \pm 31.03$ & 3,170 \\
\hline & $235-250$ & $26.68 \pm 3.41$ & $49.01 \pm 10.85$ & $137.38 \pm 19.26$ & $86.19 \pm 11.07$ & 22.7 (778) & $31.1(1070)$ & $46.2(1585)$ & $83.14 \pm 31.05$ & 3,433 \\
\hline & $251-275$ & $26.83 \pm 3.37$ & $49.54 \pm 10.66$ & $139.11 \pm 19.67$ & $87.07 \pm 11.29$ & $21.0(707)$ & $32.1(1083)$ & $46.9(1580)$ & $83.28 \pm 30.84$ & 3,370 \\
\hline & ${ }^{3} 276$ & $27.03 \pm 3.26$ & $49.72 \pm 10.07$ & $142.30 \pm 20.31$ & $88.50 \pm 11.26$ & $20.3(641)$ & $33.2(1095)$ & $46.9(1466)$ & $82.65 \pm 31.41$ & 3,152 \\
\hline & Total & $26.25 \pm 3.52$ & $47.31 \pm 11.87$ & $135.21 \pm 19.84$ & $84.84 \pm 11.41$ & $24.0(7248)$ & $29.1(8778)$ & 46.9 (14153) & $84.65 \pm 31.97$ & 30,179 \\
\hline \multirow{11}{*}{ 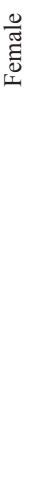 } & $<160$ & $24.34 \pm 4.58$ & $37.34 \pm 11.05$ & $122.25 \pm 18.58$ & $77.52 \pm 10.96$ & $62.0(1732)$ & $8.3(233)$ & 29.7 (829) & $88.74 \pm 34.36$ & 2,794 \\
\hline & $160-174$ & $25.06 \pm 4.68$ & $39.55 \pm 11.10$ & $124.83 \pm 19.39$ & $78.92 \pm 11.23$ & $63.8(1632)$ & $8.3(211)$ & $27.9(714)$ & $87.69 \pm 33.17$ & 2,557 \\
\hline & $175-186$ & $25.59 \pm 4.85$ & $41.87 \pm 11.39$ & $126.93 \pm 19.95$ & $80.35 \pm 11.28$ & $66.7(1744)$ & $7.7(200)$ & $25.6(669)$ & $85.67 \pm 32.01$ & 2,613 \\
\hline & $187-198$ & $25.97 \pm 5.00$ & $43.48 \pm 11.99$ & $129.72 \pm 20.94$ & $81.45 \pm 11.81$ & $69.2(1802)$ & $6.7(174)$ & $24.1(626)$ & $84.98 \pm 31.23$ & 2,602 \\
\hline & 199-209 & $26.38 \pm 4.98$ & $45.29 \pm 11.58$ & $132.15 \pm 22.58$ & $82.24 \pm 11.96$ & $68.9(1729)$ & $7.3(184)$ & $23.8(598)$ & $85.16 \pm 31.71$ & 2,511 \\
\hline & $210-221$ & $26.48 \pm 4.90$ & $46.95 \pm 11.42$ & $134.07 \pm 22.10$ & $83.53 \pm 11.68$ & 70.3 (1887) & $7.4(200)$ & $22.3(599)$ & $84.42 \pm 31.47$ & 2,686 \\
\hline & $222-234$ & $26.85 \pm 4.95$ & $48.72 \pm 11.23$ & $136.26 \pm 23.05$ & $84.38 \pm 12.11$ & $72.8(1833)$ & $7.0(178)$ & $20.2(508)$ & $82.92 \pm 30.50$ & 2,519 \\
\hline & $235-250$ & $27.33 \pm 4.93$ & $50.74 \pm 10.84$ & $139.13 \pm 22.55$ & $85.50 \pm 11.99$ & 74.8 (1903) & $6.6(167)$ & $18.6(474)$ & $84.68 \pm 30.27$ & 2,544 \\
\hline & $251-275$ & $27.33 \pm 4.70$ & $52.52 \pm 10.11$ & $141.60 \pm 22.32$ & $86.04 \pm 11.67$ & 73.7 (1925) & $6.8(177)$ & $19.5(508)$ & $84.67 \pm 30.59$ & 2,610 \\
\hline & ${ }^{3} 276$ & $27.23 \pm 4.46$ & $54.48 \pm 9.73$ & $145.54 \pm 22.82$ & $87.72 \pm 11.74$ & $73.6(1890)$ & $7.0(181)$ & $19.4(498)$ & $83.37 \pm 30.58$ & 2,569 \\
\hline & Total & $26.24 \pm 4.90$ & $46.02 \pm 12.30$ & $133.16 \pm 22.62$ & $82.72 \pm 12.05$ & 69.5 (18077) & $7.3(1905)$ & $23.2(6023)$ & $85.26 \pm 31.68$ & 26,005 \\
\hline
\end{tabular}

*BMI, Body Mass Index

lowest cholesterol decile, there was 3.3 times higher risk for non-CVD $(n=116)$ than CVD $(n=35)$. Furthermore, in females (467), considering $100 \%$ all-causes of death, $71.1 \%$ (332) were non-CVD and 28.9\% (135) were CVD, revealing approximately 2.5 times higher death rates for non-CVD compared to CVD. Moreover, regarding all-causes of death $(\mathrm{n}=36)$ in the lowest cholesterol decile, there was 2.6 times higher risk for non-CVD $(n=26)$ compared to CVD $(\mathrm{n}=10)$.

Table 3 describes age-adjusted and multivariate-adjusted hazard ratio of all-cause death and total mortality of non-CVD in different deciles of cholesterol level among males and females. There was significant inverse association between all-cause and non-CVDs deaths and cholesterol level $(<160 \mathrm{mg} / \mathrm{dl})$ after controlling age, BMI, systolic and diastolic blood pressure, and smoking status among males $(\mathrm{HR}=1.5,95 \% \mathrm{CI}$ : $1.19-1.89, \mathrm{HR}=2.06$, 95\% CI: 1.54-2.74). In females, significant inverse association existed for all-cause of death, however, this association was insignificant for non-CVD lowest cholesterol levels (HR=1.53, 95\% CI:1.01-2.34; CVD, HR $=1.52,95 \%$ CI:0.91- 2.50).

Among females, an inverse significant association was found in the fourth decile for all-cause and non-CVD deaths $(\mathrm{HR}=1.52,95 \% \mathrm{CI}: 1.06-2.18, \mathrm{HR}=1.78,95 \% \mathrm{CI}$ : 1.16-2.71).

In-depth analysis for non-CVD, Table 4 demonstrates cancer, NCLD, other non-cancer/non-CVD deaths for males and females. In males, results indicated significant risk association with lowest cholesterol $(<160 \mathrm{mg} / \mathrm{dl})$ for cancer and NCLD (HR=1.52, 95\% CI: 1.06-2.18; HR=10.73, 95\% CI: 3.74-30.18), however, with insignificant hazard ratios for other non-cancer/non-CVD (HR=1.84, 95\%CI: 0.98-3.47). In the second decile of NCLD death and third decile of other non-cancer/non-CVD, results were significantly associated with low cholesterol risk for males ( $\mathrm{HR}=3.73$, 95\% CI: 1.16-11.95; HR=2.05 95\% CI :( 1.12, 3.75). In females, only NCLD indicates significant risk association with lowest cholesterol decil e $(\mathrm{HR}=25.8$, 95\% CI: 3.09-217.70).

\section{Discussion}

The primary objective of this study was to determine if an association exists between excess non-CVD mortality and low serum cholesterol. The study populations were Italians aged between 20 to 69 years old. The participants were followed over a 9-year period. Subjects were selected similar in health status for sociodemographic, clinical, anthropometric, and laboratory variables. Findings showed a "J" shaped curve for all-causes and total non-CVD mortality across cholesterol level deciles which remains after controlling for confounders . Most of the excess mortality at the first decile of cholesterol level $(<160 \mathrm{mg} / \mathrm{dl})$ belonged to non-CVD deaths. Interpretation of the inverse association between cholesterol level and non-CVD mortality was complex. 


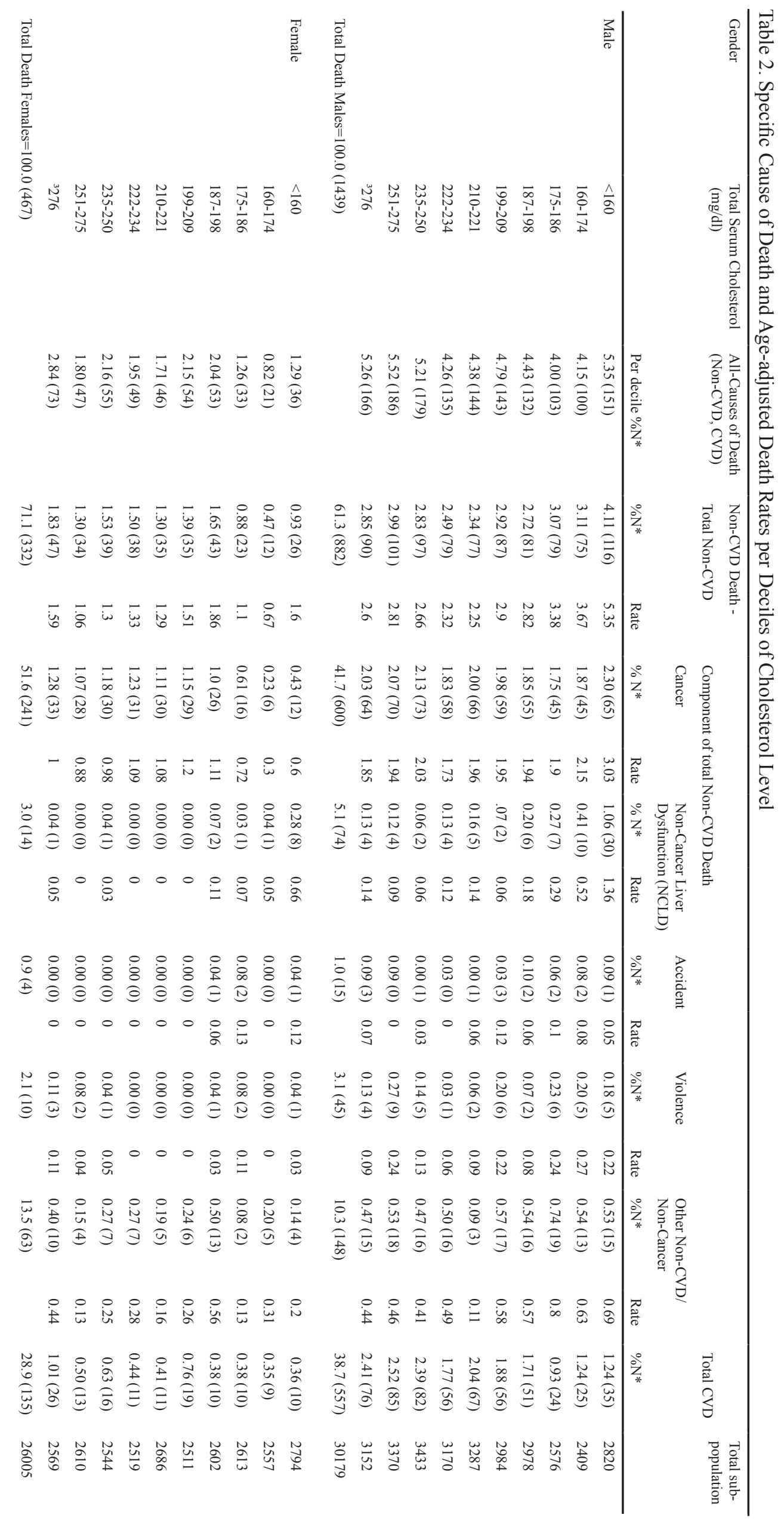


DOI:10.31557/APJCP.2019.20.5.1361

Low Serum Cholesterol and Non-Cardiovascular Mortality in Male and Female Population

Table 3. Age-adjusted and Multivariate-adjusted Hazard Ratio for All Causes of death and total non-CVD Mortality of Different Cholesterol Levels for Males and Females

\begin{tabular}{|c|c|c|c|c|c|c|c|}
\hline \multirow[t]{3}{*}{ Gender } & \multirow{3}{*}{$\begin{array}{l}\text { Total Serum } \\
\text { Cholesterol (mg/dl) }\end{array}$} & \multicolumn{3}{|c|}{ All Causes of Death (Non-CVD,CVD) } & \multicolumn{3}{|c|}{ Total Non-CVD } \\
\hline & & $\mathrm{N}^{*}$ & Age-adj** & Multivariate-adj*** & $\mathrm{N}^{*}$ & Age-adj** & Multivariate-adj*** \\
\hline & & & Hazard Ratio & Hazard Ratio $95 \%(\mathrm{CI})$ & & Hazard Ratio & Hazard Ratio $95 \%(\mathrm{CI})$ \\
\hline \multirow[t]{10}{*}{ Male } & $<160$ & 151 & 1.37 & $1.50(1.19,1.89)$ & 116 & 1.98 & $2.06(1.54,2.74)$ \\
\hline & $160-174$ & 100 & 0.92 & $1.01(0.76,1.30)$ & 75 & 1.29 & $1.36(0.99,1.87)$ \\
\hline & $175-186$ & 103 & 0.86 & $0.93(0.72,1.20)$ & 79 & 1.24 & $1.30(0.95,1.78)$ \\
\hline & $187-198$ & 132 & 0.9 & $0.97(0.76,1.22)$ & 81 & 1.07 & $1.10(0.81,1.51)$ \\
\hline & $199-209$ & 143 & 0.98 & $1.05(0.84,1.33)$ & 87 & 1.15 & $1.19(0.87,1.61)$ \\
\hline & $210-221$ & 144 & 0.87 & $0.92(0.73,1.16)$ & 77 & 0.9 & $0.92(0.67,1.26)$ \\
\hline & $222-234$ & 135 & 0.8 & $0.84(0.67,1.06)$ & 79 & 0.93 & $0.95(0.69,1.29)$ \\
\hline & $235-250$ & 179 & 1.01 & $1.05(0.84,1.30)$ & 97 & 1.06 & $1.08(0.80,1.44)$ \\
\hline & $251-275$ & 186 & 1.02 & $1.06(0.85,1.31)$ & 101 & 1.05 & $1.07(0.79,1.43)$ \\
\hline & $\left(\mathrm{RG}^{* * * *}\right)-^{3} 276$ & 166 & 1 & $1.00(---)$ & 90 & 1 & $1.00(---)$ \\
\hline Total Males & & 1,439 & & & 882 & & \\
\hline \multirow[t]{10}{*}{ Female } & $<160$ & 36 & 1.46 & $1.53(1.01,2.34)$ & 26 & 1.46 & $1.52(0.91,2.50)$ \\
\hline & $160-174$ & 21 & 0.69 & $0.74(0.44,1.25)$ & 12 & 0.6 & $0.62(0.32,1.21)$ \\
\hline & $175-186$ & 33 & 0.95 & $1.01(0.65,1.54)$ & 23 & 1.01 & $1.04(0.62,1.74)$ \\
\hline & $187-198$ & 53 & 1.44 & $1.52(1.06,2.18)$ & 43 & 1.72 & $1.78(1.16,2.71)$ \\
\hline & $199-209$ & 54 & 1.31 & $1.36(0.95,1.96)$ & 35 & 1.29 & $1.32(0.84,2.06)$ \\
\hline & $210-221$ & 46 & 1.01 & $1.04(0.72,1.52)$ & 35 & 1.13 & $1.16(0.74,1.81)$ \\
\hline & $222-234$ & 49 & 0.94 & $0.97(0.67,1.42)$ & 38 & 1.06 & $1.08(0.69,1.70)$ \\
\hline & $235-250$ & 55 & 0.91 & $0.96(0.66,1.40)$ & 39 & 0.97 & $0.99(0.64,1.54)$ \\
\hline & $251-275$ & 47 & 0.67 & $0.69(0.47,1.02)$ & 34 & 0.79 & $0.81(0.51,1.26)$ \\
\hline & $\left(\mathrm{RG}^{* * * * *}\right)^{3} 276$ & 73 & 1 & $1.00(---)$ & 47 & 1 & $1.00(---)$ \\
\hline Total Females & & 467 & & & 332 & & \\
\hline
\end{tabular}

*N, number of subjects;**Adj., adjusted; ***Multivariate-adjusted for (age, body-mass index, systolic blood pressure, diastolic blood pressure, and smoking status; $* * * * \mathrm{RG}$, Reference Group.

In some studies, the inverse association was attenuated or disappeared after excluding early deaths within 1-5 years from baseline, suggesting that the inverse association was related to preclinical conditions which caused lower cholesterol concentrations (Bowe et al., 2016; Törnberg et al., 1989). There is arguably sufficient biologic and epidemiologic rationale to warrant the inference that low serum cholesterol and excess mortality from non-CVD is causal, even though other important confounding factors and/or unmeasured factors may exist. Similar to this study, previous studies showed that inverse association persisted over the longer follow-up, even when the first 5-year deaths (Schatzkin et al., 1987; Garcia-Palmier et al., 1981) the first 10-year deaths were excluded (Frank et al.,1991; Smith et al.,1990). Some of these studies indicated that persistent inverse associations were attenuated when covariates such as socioeconomic and other laboratory factors were taken into account. These findings suggested that the inverse associations were due to other risk factors which elevated the risk of death. The present analysis in line with other studies; however, showed a persistent inverse association even after adjusting confounding and interaction factors. Furthermore, after age-adjusted and multivariate-adjusted hazard ratio for all-cause of
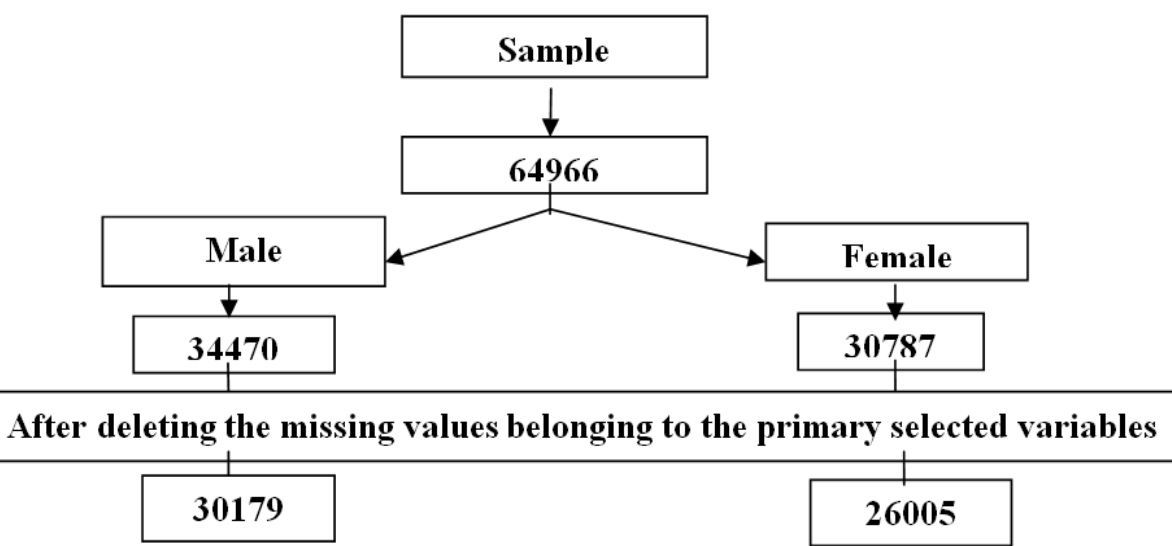

Figure 1. Sample Size Follow-up Chart for Male and Female Population Extracted from RIFLE Studies 
Table 4. Age-adjusted and Multivariate-adjusted Hazard Ratio for Cancer, Non-cancer Liver Dysfunction, and Other Non-cancer/Non-cardiovascular diseaseCVD Deaths for Different Cholesterol Levels for Males and Females

\begin{tabular}{|c|c|c|c|c|c|c|c|c|c|c|}
\hline \multirow{4}{*}{ Gender } & \multirow{4}{*}{$\begin{array}{l}\text { Total Serum } \\
\text { Cholesterol } \\
(\mathrm{mg} / \mathrm{dl})\end{array}$} & \multirow{2}{*}{\multicolumn{3}{|c|}{ Cancer }} & \multirow{2}{*}{\multicolumn{3}{|c|}{ Non-cancer Liver Dysfunction }} & \multicolumn{3}{|c|}{ NON-CVD } \\
\hline & & & & & & & & \multicolumn{3}{|c|}{ Other Non-cancer/Non-CVD } \\
\hline & & \multirow[t]{2}{*}{$\mathrm{N}^{*}$} & Age-adj** & Multivariate-adj*** & $\mathrm{N}^{*}$ & Age-adj** & Multivariate-adj*** & $\mathrm{N}^{*}$ & Age-adj** & Multivariate-adj*** \\
\hline & & & $\begin{array}{c}\text { Hazard } \\
\text { Ratio }\end{array}$ & $\begin{array}{c}\text { Hazard Ratio } \\
95 \%(\mathrm{CI})\end{array}$ & & $\begin{array}{c}\text { Hazard } \\
\text { Ratio }\end{array}$ & $\begin{array}{l}\text { Hazard Ratio } 95 \% \\
\text { (CI) }\end{array}$ & & $\begin{array}{c}\text { Hazard } \\
\text { Ratio }\end{array}$ & $\begin{array}{c}\text { Hazard Ratio } 95 \% \\
\text { (CI) }\end{array}$ \\
\hline \multirow[t]{10}{*}{ Male } & $<160$ & 65 & 1.47 & $1.52(1.06,2.18)$ & 30 & 11.16 & $10.73(3.74,30.18)$ & 21 & 1.74 & $1.84(0.98,3.47)$ \\
\hline & $160-174$ & 45 & 1.02 & $1.08(0.72,1.60)$ & 10 & 3.9 & $3.73(1.16,11.95)$ & 20 & 1.66 & $1.77(0.93,3.36)$ \\
\hline & $175-186$ & 45 & 0.98 & $1.03(0.70,1.52)$ & 7 & 2 & $2.05(0.58,7.29)$ & 27 & 1.95 & $2.05(1.12,3.75)$ \\
\hline & $187-198$ & 55 & 1.02 & $1.05(0.73,1.51)$ & 6 & 1.46 & $1.44(0.38,5.36)$ & 20 & 1.8 & $1.23(0.64,2.34)$ \\
\hline & $199-209$ & 59 & 1.06 & $1.10(0.77,1.58)$ & 2 & 0.5 & $0.57(0.10,3.11)$ & 26 & 1.56 & $1.64(0.89,2.99)$ \\
\hline & $210-221$ & 66 & 1.05 & $1.08(0.76,1.53)$ & 5 & 1.24 & $1.22(0.33,4.55)$ & 6 & 0.32 & $0.34(0.13, .85)$ \\
\hline & $222-234$ & 58 & 0.93 & $0.95(0.66,1.36)$ & 4 & 1.01 & $0.98(0.24,3.90)$ & 17 & 0.91 & $0.94(0.49,1.83)$ \\
\hline & $235-250$ & 73 & 1.08 & $1.10(0.78,1.55)$ & 2 & 0.46 & $0.45(0.08,2.46)$ & 22 & 1.11 & $1.14(0.62,2.13)$ \\
\hline & $251-275$ & 70 & 1.01 & $1.04(0.73,1.46)$ & 4 & 0.92 & $0.90(0.23,3.62)$ & 27 & 1.2 & $1.24(0.67,2.27)$ \\
\hline & $\left(\mathrm{RG}^{* * * *}\right)^{3} 276$ & 64 & 1 & $1.00(---)$ & 4 & 1 & $1.00(---)$ & 22 & 1 & $1.00(---)$ \\
\hline \multicolumn{2}{|c|}{ Total Males } & 600 & & & 74 & & & 208 & & \\
\hline \multirow[t]{10}{*}{ Female } & $<160$ & 12 & 0.99 & $1.03(0.52,2.05)$ & 8 & 27.61 & $25.80(3.09,217.70)$ & 6 & 1.12 & $1.16(0.42,3.17)$ \\
\hline & $160-174$ & 6 & 0.39 & $0.41(0.16,1.06)$ & 1 & 3.27 & $2.54(0.16,41.60)$ & 5 & 0.88 & $0.89(0.32,2.57)$ \\
\hline & $175-186$ & 16 & 0.98 & $1.02(0.55,1.90)$ & 1 & 2.66 & $2.40(0.15,39.00)$ & 6 & 0.9 & $0.92(0.34,2.46)$ \\
\hline & $187-198$ & 26 & 1.5 & $1.56(0.92,2.65)$ & 2 & 4.33 & $3.76(0.34,42.21)$ & 15 & 2 & $2.01(0.94,4.28)$ \\
\hline & $199-209$ & 29 & 1.58 & $1.63(0.98,2.72)$ & 0 & 0 & $0.00(0.00)$ & 6 & 0.63 & $0.63(0.22,1.77)$ \\
\hline & $210-221$ & 30 & 1.4 & $1.45(0.87,2.40)$ & 0 & 0 & $0.00(0.00)$ & 5 & 0.54 & $0.54(0.19,1.52)$ \\
\hline & $222-234$ & 31 & 1.26 & $1.290(0.77,2.18)$ & 0 & 0 & $0.00(0.00)$ & 7 & 0.66 & $0.65(0.24,1.70)$ \\
\hline & $235-250$ & 30 & 1.13 & $1.18(0.71,1.96)$ & 1 & 1.29 & $1.22(0.07,19.55)$ & 8 & 0.55 & $0.54(0.20,1.43)$ \\
\hline & $251-275$ & 28 & 0.95 & $0.97(0.58,1.62)$ & 0 & 0 & $0.00(0.00)$ & 6 & 0.49 & $0.49(0.18,1.28)$ \\
\hline & $\left(\mathrm{RG}^{* * * *}\right)^{3} 276$ & 33 & 1 & $1.00(---)$ & 1 & 1 & $1.00(---)$ & 13 & 1 & $1.00(---)$ \\
\hline \multicolumn{2}{|c|}{ Total Females } & 241 & & & 14 & & & 77 & & \\
\hline
\end{tabular}

*N, number of subjects; **Adj., adjusted; ***Multivariate-adjusted for (age, body-mass index, systolic blood pressure, diastolic blood, pressure, and smoking status; ****RG, Reference Group

death and total non-CVD mortality inverse significant associations for lowest cholesterol for males exists. The inverse significant associations between low cholesterol level $(<160 \mathrm{mg} / \mathrm{dl})$ and all-causes of death and marginally non-CVD mortality were found for the female population.

In females, results showed an inverse significant association between the lowest cholesterol deciles and all-cause of death, non-cancer liver dysfunction, and marginally non-CVD mortality. However, other noncancer/non-CVD total mortality showed an insignificant risk. Females naturally store more fatty tissue than males. This physiological difference may be more protective against excess mortality events, with longer life expectancy among females than males. These differences were distinguishable in statistical analysis, from the descriptive to analytical phase.

Study findings suggested different cholesterol levels for various subgroups in the male population had different biological meanings for those in the low serum cholesterol group . The lowest decile $(<160 \mathrm{mg} / \mathrm{dl})$ indicated relatively high hazard ratio for all-cause of death, total non-CVD, cancer, and non-cancer liver dysfunction for the male population and all-cause and total non-cancer liver dysfunction in females. These findings indicated that a decrease towards the lowest decile $(<160 \mathrm{mg} / \mathrm{dl})$ had a different biological magnitude than towards the other decile groups. This information suggested the indicative properties of cholesterol in the level of the cell membrane.

Moreover, outcomes remained statistically significant after controlling for age, BMI, systolic diastolic blood pressure, and cigarette smoking status. The findings of this study are consistent with the results of a metaanalysis on 19 cohort studies conducted on Caucasian (Iso etal.,1989; Neaton etal.,1992 ) and American-Japanese populations, 24-25 reporting a "U", "J" or "L"-shaped pattern between total cholesterol and total mortality in men (Reed etl.,1986; Martin etal.,1986). This pattern is composed of a positive association between blood cholesterol and increased CVD death (clearly a clinical and public health significance), and an inverse association between cholesterol and excess mortality due to non-CVD (cancer and non-cancer, etc) deaths. Based upon the results of previous studies regarding age effect ( $>50$ years) for association between low cholesterol and non-CVD death, there was no need in this study to look for age as a separate dominant predictor factor for non-CVD death, even though interactions and confounding were controlled in this study. Consequently, after multivariate-adjusting for age, BMI, systolic and diastolic blood pressures, and smoking status, cholesterol levels for all-cause of death in the first decile were significantly associated with risk of mortality in both genders. Clear-cut non-CVD death rates 
at low cholesterol levels $<160 \mathrm{mg} / \mathrm{dl}$ were demonstrated in this study. Analytical results of this study placed strong emphasis on medical and pharmacological management of low cholesterol, specifically the effects of the lowest cholesterol level on non-CVD in the male population.

\section{Strengths and Limitations}

As in any observational cohort study, these findings may be confounded by unmeasured variables. However, we did our best to minimize this limitation by gathering fairly accurate data by the same center. In addition, standardization and quality control of measurements were observed.

An attempt was made to identify a low cholesterol risk factor for mortality in this analysis and may not be generalizable to other populations other than study ones due to unknown or unmeasured factors, or low cardiovascular death. Although this study may have an underestimation of risk factors in the target population, reporting biases were thought to be minimal.

Despite any study biases, these findings demonstrated a strong association between cholesterol risk factors as a representative of significant morbidity, mortality in different cholesterol levels specifically lowest cholesterol decile and non-CVD death status.

Nevertheless, this study, like others may have some limitations. The lack of information on post-treatment recur $\neg$ rence and the change of total cholesterol levels are the limitations of current study. A prospective study is required to determine the prognostic and treat $\neg$ ment value of cholesterol.

In conclusion, findings of this study indicated a significant inverse association between lowest cholesterol level and all-causes of death, non-CVD death for males and females.

\section{Abbreviations}

Non-CVD: Non-cardiovascular disease, CVD: Cardiovascular disease, CI: Confi $\neg$ dence intervals, NCLD: Non-cancer liver dysfunction, CHD: Coronary heart disease, NIM: Non-illness mortality, NIH: National Institute of Health, RIFLE: Risk Factors of Life Expectancy, ICD: International Classification of Diseases, WHO: World Health Organization, BMI: Body mass index, HR: Hazard ratio, ADJ: Adjusted, RG: Reference Group.

\section{Authors' contributions}

Nader Parsa and Maurizio Trevisan were responsible for the concept and the design of the study, Nader Parsa carried out the development of the model, analysis of data, and interpretation of results. Nader Parsa with Samira Taravatmanesh cooperated in its design and helped draft the manuscript. All authors read and approved the final manuscript.

\section{Consent for publication \\ Not applicable.}

Ethics approval and consent to participate

The study was approved by the New York State University School of Medicine at Buffalo, by permission of Professor M. Trevisan. Written informed consent was obtained Risk Factors and Life Expectancy (RIFLE) project.

\section{Disclaimers}

Authors state that views expressed in the submitted manuscript are their own work.

\section{Competing interests}

The authors declared no conflict of interests.

\section{Acknowledgments}

The authors would like to thank RIFLE.

\section{References}

Auerbach BJ, Parks JS (1989). Lipoprotein abnormalities associated with lipopolysaccharide-induced lecithin: cholesterol acyltransferase and lipase deficiency. $J$ Biol Chem, 264, 10264-70.

Bowe B, Xie Y, Xian H, et al (2016). High density lipoprotein cholesterol and the risk of all-cause mortality among US veterans. Clin J Am Soc Nephrol, 11, 1784-93.

Cambien F, Ducimetiere P, Richard J (1980). Total serum cholesterol and cancer mortality in a middle-aged male population. Am J Epidemiol, 112, 388-94.

Ettinger WH, Miller LD, Albers JJ, Smith TK, Parks JS (1990). Lipopolysaccharide and tumor necrosis factor cause a fall in plasma concentration of lecithin: cholesterol acyltransferase in cynomolgus monkeys. J Lipid Res, 31, 1099-1107.

Frank JW, Reed DM, Grove JS, Benfante R (1992). Will lowering population levels of serum cholesterol affect total mortality? Expectations from the Honolulu Heart Program. J Clin Epidemiol, 45, 333-46.

Garcia P, Sorlie PD, Costas Jr R, Havlik RJ (1981). An apparent inverse relationship between serum cholesterol and cancer mortality in Puerto Rico. Am J Epidemiol, 114, 29-40.

Holme I (1991). An analysis of randomized trials evaluating the effect of cholesterol reduction on total mortality and coronary heart disease incidence. Circulation, 84, 2610-11.

Hasegawa A, Kojima F, Ueda M, Tanaka Y, Nitta K (2016). Triglyceride to high-density lipoprotein cholesterol ratio predicts cardiovascular events in maintenance hemodialysis patients. Ren Replace Therap, 2, 60.

Iso H, Jacobs Jr DR, Wentworth D, et al (1989). Serum cholesterol levels and six-year mortality from stroke in 350,977 men screened for the multiple risk factor intervention trial. $N$ Engl J Med, 320, 904-10.

Jacobs D, Blackburn H, Higgins,M, et al (1992). Report of the conference on low blood cholesterol: mortality associations. Circulation, 86, 1046-60.

Kim JM, Stewart R, Kang HJ, et al (2014). Longitudinal associations between serum cholesterol levels and suicidal ideation in an older Korean population. $J$ Affect Disord, 152, 517-21.

Krycer JR, Brown AJ (2013). Cholesterol accumulation in prostate cancer: a classic observation from a modern perspective. Biochim Biophys Acta, 1835, 219-29.

Martin M, Browner W, Hulley S, Kuller L, Wentworth D (1986). Serum cholesterol, blood pressure, and mortality: 
implications from a cohort of 361,662 men. Lancet, 328, 933-6.

Neaton JD, Blackburn H, Jacobs D, et al (1992). Serum cholesterol level and mortality findings for men screened in the Multiple Risk Factor Intervention Trial. Arch Int Med, 152, 1490-1500.

Parsa N, Taravatmanesh S, Trevisan M (2017). Comparison of noncardiovascular and cardiovascular mortality related to low serum cholesterol among males and females. Iran Red Crescent Med J, 19, e14369.

Parsa N, Taravatmanesh S, Trevisan M (2018). Is low cholesterol a risk factor for cancer mortality?. Eur J Cancer Prev, 27, 570-6.

Reed D, Yano K, Kagan A (1986). Lipids and lipoproteins as predictors of coronary heart disease, stroke, and cancer in the Honolulu Heart Program. Am J Med, 80, 871-8.

Sahebzamani FM, D'Aoust RF, Friedrich D, et al (2013). Relationship among low cholesterol levels, depressive symptoms, aggression, hostility, and cynicism. J Clin Lipidol, 7, 208-16.

Schatzkin A, Taylor P, Carter C, et al (1987). Serum cholesterol and cancer in the NHANES I epidemiologic followup study. Lancet, 330, 298-301.

Smith GD, Shipley MJ, Marmot MG, Rose G (1992). Plasma cholesterol concentration and mortality: the Whitehall Study. JAMA, 267, 70-6.

Tanaka T, Okamura T (2012). Blood cholesterol level and risk of stroke in community-based or worksite cohort studies: a review of Japanese cohort studies in the past 20 years. Keio J Med, 61, 79-88.

Törnberg SA, Holm LE, Carstensen JM, Eklund GA (1989). Cancer incidence and cancer mortality in relation to serum cholesterol. J Natl Cancer Inst, 81, 1917-21.

Verdery RB, Goldberg AP (1991). Hypocholesterolemia as a predictor of death: a prospective study of 224 nursing home residents. J Gerontol, 46, 84-90.

World Health Organization (2010). International statistical classification of diseases and related health problems (Vol. 1). World Health Organization.

\section{(ब) $(\mathbb{2}$}

This work is licensed under a Creative Commons AttributionNon Commercial 4.0 International License. 